\title{
INFLUÊNCIA DO MÉTODO DE DEFUZZIFICAÇÃO EM MENSURAÇÕES COM CONTROLADORES FUZZY
}

\author{
Gabriela Carré dos Santos \\ Universidade Federal do Rio Grande, Av. Itália, km 8, Bairro Carreiros, Rio Grande/RS \\ gabrielacarre.s@gmail.com
}

Paulo Siga Thomaz

Universidade Federal do Rio Grande, Av. Itália, km 8, Bairro Carreiros, Rio Grande/RS paulosigathomaz@gmail.com

Franciele Muller Ribeiro

Universidade Federal do Rio Grande, Av. Itália, km 8, Bairro Carreiros, Rio Grande/RS francielemullerribeiro@gmail.com

Jéssica Fernandes Araújo

Universidade Federal do Rio Grande, Av. Itália, km 8, Bairro Carreiros, Rio Grande/RS jessicaf.araujo1@gmail.com

Viviane Leite Dias de Mattos

Universidade Federal do Rio Grande, Av. Itália, km 8, Bairro Carreiros, Rio Grande/RS viviane.leite.mattos@gmail.com

\section{Resumo}

A mensuração de um constructo que represente o pensamento humano é muito difícil de ser executada sob o enfoque da lógica clássica onde só existem dois graus de verdade. Neste contexto ganha importância a lógica fuzzy que admite várias graduações entre o puramente verdadeiro e o puramente falso. Este trabalho, desenvolvido como estudo piloto, compara o desempenho de alguns métodos de defuzzificação (última etapa dos controladores fuzzy) utilizados em instrumento que mensura a satisfação. Participaram deste estudo piloto 16 indivíduos. As informações obtidas foram defuzzificadas com o uso de três métodos, sendo os resultados comparados por uma Análise de Variância complementada pelo alfa de Cronbach. Não foram encontradas evidências de diferença entre os métodos, mas o método da Média dos Máximos apresentou confiabilidade levemente superior aos demais.

Palavras-Chaves: Controladores Fuzzy; Método de Defuzzificação; Satisfação; Qualidade.

\begin{abstract}
Abstrac
The measurement of a construct representing human thought is very difficult to be performed by classical logic where there are only two degrees of truth. Fuzzy logic gains importance in this context because it admits many gradations between the purely truth and purely false. This study was developed as a pilot study and compares the performance of some methods of defuzzification (last step of fuzzy controllers) at an instrument that measures the satisfaction. Sixteen individuals participated in this pilot study. The information obtained was defuzzificated using three methods. The results were compared by analysis of variance, complemented by Cronbach's alpha. It wasn't found evidence of difference between the methods, but the Mean Maximum Method showed slightly higher than the other reliability.
\end{abstract}

Keywords: Fuzzy Controllers; Defuzzification Method; Satisfaction; Quality. 


\section{INTRODUÇÃO}

O sucesso de qualquer análise estatística depende muito da qualidade da mensuração dos dados utilizados, ou melhor, da fidedignidade da escala de mensuração utilizada. Dentro de um enfoque clássico, existem quatro tipos de escalas: nominal, ordinal, intervalar e de razão, sendo as duas primeiras qualitativas e as demais quantitativas. Entretanto, quando utilizadas sob o enfoque da lógica clássica, algumas podem deixar a desejar pela sua falta de precisão, enquanto que outras pelo seu rigor.

Para contornar este problema, em determinados contextos pode ser extremamente pertinente a utilização da lógica fuzzy que se baseia nos conceitos da teoria dos conjuntos fuzzy proposta por Zadeh em 1965. Neste caso, em escalas qualitativas, existe uma graduação de graus de verdade variando entre o pertence e o não pertence, reduzindo a perda de informação e refletindo melhor a realidade. Um elemento pode pertencer, não pertencer ou estar parcialmente presente em uma categoria, sendo associado a ele um grau de pertinência que informa o quanto ele pertence a esta categoria. Já nas escalas quantitativas um resultado discreto não é tratado como exatamente igual ao valor obtido, mas como o entorno deste valor. Em ambos os casos a mensuração se aproximará mais da forma de pensar dos indivíduos.

Um procedimento que tem sido bastante utilizado na mensuração de variáveis, conforme relatado em Souto (2005), Abadi e Khooban (2013) e Sanaye, Dehghandokht e Farjat (2012), entre outros, são os controladores fuzzy, desenvolvidos em três estágios: fuzzyficação das entradas discretas, processamento através de regras de inferência fuzzy e cálculo de saídas discretas por defuzzificação. Vários métodos já foram propostos para a execução de cada um destes estágios e o presente estudo compara o desempenho de alguns métodos de defuzzificação disponíveis na literatura a serem utilizados em instrumento que mensura a satisfação. Trata-se de uma etapa do processo de validação e aferição do referido instrumento para identificar o método a ser utilizado.

\section{FUNDAMENTAÇÃO TEÓRICA}

A mensuração de variáveis por meio de controladores fuzzy é feita mediante a desconstrução do constructo para que a avaliação seja feita de maneira mais pontual e específica, o que poderia resultar em maior precisão. Na mensuração de variáveis subjetivas é bastante usual dividi-las sucessivamente até encontrar uma unidade de mensuração satisfatória.

Antunes (2009) e Rosa (2012), por exemplo, avaliaram o risco em, respectivamente, auditorias contábeis e avaliações de cursos de graduação, dividindo-os em classes, fatores e elementos de avaliação. Nestes estudos, os elementos (unidade mensuradas) foram avaliados por meio de valores discretos e transformados em valores categóricos com diferentes graus de pertinência aos conjuntos fuzzy, o que se caracteriza como processo de fuzzificação, o primeiro estágio do controlador fuzzy. Os graus de pertinência são definidos por funções de pertinência que podem ser representadas por diferentes funções matemáticas. A mais encontrada na literatura é a função triangular.

No segundo estágio, as entradas fuzzificadas são combinadas originando um resultado fuzzy para cada fator, o que posteriormente é feito para as classes, originando um resultado fuzzy para cada classe. Este processo, denominado inferência, usa regras definidas por especialistas que descrevem as diversas possibilidades de raciocínio. Dubois (1980) designou-as de Agregação ou Resolução de Conflitos. Elas se baseiam na aplicação de regras de controle, que podem ser de caráter condicional (se ... então...) ou incondicional (se), realizando somente uma asserção e podendo conter mais de um condicionante. A relação entre as premissas das regras se dá por meio de conectivos lógicos - operador de junção (e) ou de disjunção (ou). $\mathrm{O}$ método mais citado na literatura por ter sido usado com esta finalidade é o Método de Mamdani ou Mínimo/Máximo. 
No último estágio os resultados fuzzy encontrados são defuzzificados, sendo transformados em valores discretos. Vários métodos podem ser utilizados e os mais citados na literatura são descritos a seguir.

Método dos Máximos: Este método (MoM) transforma a saída fuzzy em saída discreta identificando o core do conjunto fuzzy ao qual o elemento pertence com maior grau de pertinência. Se o valor máximo não for único, considera-se o ponto central do intervalo de cores.

Método Média dos Máximos: Este método (CoM) transforma a saída fuzzy em saída discreta encontrando a média aritmética ponderada entre os cores dos conjuntos fuzzy às quais o elemento pertence, ponderadas pelo grau de pertinência, conforme equação (1).

$$
Y=\frac{\sum_{i=1}^{k} \mu_{i} \cdot Y_{i}}{\sum_{i=1}^{k} \mu_{i}}
$$

onde: $Y$ é a saída discreta; $k$ é a quantidade de conjuntos fuzzy a que o elemento pertence; $\mu_{i}$ é o grau de pertinência com que o elemento pertence ao i-ésimo conjunto fuzzy; $Y_{i}$ é o core do $i$-ésimo conjunto fuzzy a que o elemento pertence. Considera-se core de um conjunto fuzzy $N$ consiste de todos os elementos $x$ cujo grau de pertinência é igual a 1, ou seja:

$$
\operatorname{Core}(N)=\left\{y \in I \mid \mu_{N}(y)=1\right\}
$$

Método do Centróide: Este método (CoA) transforma a saída fuzzy em saída discreta encontrando a média aritmética entre os centros de gravidade dos conjuntos fuzzy aos quais o elemento pertence, ponderados pelo grau de pertinência, conforme equação (2).

$$
Y=\frac{\sum_{i=1}^{k} \mu_{i} \cdot Y_{i}}{\sum_{i=1}^{k} \mu_{i}}
$$

onde: $Y$ é a saída discreta; $k$ é a quantidade de conjuntos fuzzy a que o elemento pertence; $\mu_{i}$ é o grau de pertinência com que o elemento pertence ao $i$-ésimo conjunto fuzzy; $Y_{i}$ é o centro de gravidade do i-ésimo conjunto fuzzy a que o elemento pertence.

A principal desvantagem desse método é que ele se torna difícil de ser executado com funções de pertinência complexas, já que o centróide das áreas não é encontrado facilmente. Alguns autores consideram que muitas das implementações CoA são apenas aproximações, uma vez que negligenciam sobreposição de áreas.

Vários estudos, alguns dos quais citados a seguir, relatam a utilização de controladores fuzzy com sucesso.

Godoy, Franceschetti e Bose (1999) desenvolveram um sistema para geração de energia eólica capturada por uma turbina de eixo vertical, usando três controladores fuzzy. O primeiro tinha a função de achar a velocidade de vento ideal do gerador, otimizando a eficiência aerodinâmica e assim alcançando a potência máxima na rede. O segundo ajustava o melhor nível de fluxo rotórico no gerador de indução, reduzindo-o para valores menores que o normal, diminuindo as perdas de ferro no núcleo da máquina e aumentando o rendimento global. O terceiro controlador realizava uma malha de controle geral de velocidade do gerador, contra turbulências, vórtices do vento e torque oscilatório da turbina, tornando o sistema eólico imune às ressonâncias típicas das turbinas de eixo vertical. As funções de pertinência utilizadas foram triangulares e assimétricas. As regras foram baseadas na velocidade e em como sua oscilação influenciava na potência de saída do gerador e o método de defuzzificação não foi citado. Os autores observaram uma melhora em 35,5\% na operação do primeiro controlador e 8,6\% no segundo. Os controladores proporcionaram uma insensibilidade à variação de parâmetros, rápida convergência e aceitação de sinais ruidosos e 
imprecisos, causando melhoria no desempenho do sistema e proporcionando potência máxima para a rede elétrica. O sistema foi considerado como apresentando desempenho excelente.

Souto (2005) desenvolveu um sistema especialista para gerenciar o funcionamento de uma usina nuclear usando lógica fuzzy na definição de faixas de desempenho para indicadores. Nestes contextos o monitoramento rigoroso é essencial, não só para assegurar o elevado desempenho, mas também para garantir a segurança dos trabalhadores e das populações ao redor, pois existe um grande risco em potencial. O controlador fuzzy utilizou função triangular para fuzzificação, o método Mamdani para as regras de inferência e o método da média dos máximos para defuzzificação. $\mathrm{O}$ autor constatou que os resultados obtidos foram coerentes, demonstrando com elevada precisão o desempenho da usina e evidenciando a eficiência da utilização da lógica fuzzy na presença de informações imprecisas.

Abadi e Khooban (2013) utilizaram controladores fuzzy para rastreamento de trajetória de robôs móveis. Um modelo de robô virtual foi criado e os parâmetros de entrada e saída foram otimizados. Nos controladores fuzzy foi utilizada função de pertinência gaussiana para fuzzificação, o método de Mamdani para as regras de inferência e o método do centróide para defuzzificação. Os autores chegaram a conclusão que o controlador utilizado é recomendado para monitoramento integrado cinemático e dinâmico na presença de incertezas paramétricas e não paramétricas.

Sanaye, Dehghandokht, Farjat (2012) utilizaram controladores fuzzy para estudar o conforto térmico em uma cabine de um automóvel. Depois de modelar o sistema de tratamento de ar no interior da cabine, foi utilizado um controlador fuzzy com dois parâmetros - a velocidade de saída de ar do ventilador e a percentagem de ar em circulação - para controlar a temperatura do ar no interior da cabine. Usaram função de pertinência triangular, regras de inferência baseadas no método dos mínimos e máximos. Os resultados mostraram que quando se utiliza um controlador fuzzy, o controle da temperatura e ventilação no interior da cabine foi conseguido mais rapidamente o que reduziu de maneira substancial o consumo de combustível.

\section{MÉTODO}

Este trabalho é de natureza exploratória e utilizou um instrumento proposto para mensurar a satisfação usando controladores fuzzy (MATTOS et al., 2012; VITICOSKI et al., 2013) que apresenta três classes subdivididas em um, dois e quatro fatores que foram subdivididos em dois, três ou quatro elementos de avaliação, conforme quadro 1.

Os dados coletados foram fuzzificados com uma função de pertinência triangular, em uma escala que considerou cinco categorias, conforme figura 1. As regras de inferência foram definidas por especialista e seguiram o método de Mamdani. Para defuzzificação foram usados os Métodos do Centróide, do Máximo e da Média dos Máximos.

Os dados obtidos no levantamento foram analisados por meio de uma análise de variância com duas variáveis independentes: método de defuzzificação (CoM, MoM e CoA) e classe de avaliação (veículo, serviço e pontos de parada). Ao serem encontradas evidências de diferenças, um teste para comparações múltiplas (teste de Tukey) foi utilizado com o objetivo de localizá-las. O coeficiente de determinação e uma análise de resíduos avaliam a adequação da técnica utilizada. Finalizando, foi realizada uma análise da confiabilidade do instrumento por meio do alfa de Cronbach padronizado com o objetivo de conhecer a influência do método de defuzzificação na confiabilidade do instrumento.

Para a extração da amostra que forneceu os dados utilizados neste estudo foi considerada uma população alvo formada pelos estudantes que freqüentam um campus de uma instituição de ensino superior. Foram selecionados aleatoriamente um dia da semana, um pavilhão e uma sala de aula. Por conveniência foi escolhido um turno, sendo identificada uma turma: a amostra foi formada pelos alunos matriculados nesta turma e que utilizavam o transporte coletivo. 
Quadro 1 - Segregação do construto satisfação

\begin{tabular}{|c|c|c|c|}
\hline Componentes & Classes & Fatores & Elementos de avaliação \\
\hline \multirow{19}{*}{ Empresa } & \multirow{8}{*}{ Recursos } & \multirow{4}{*}{ Conforto } & Comodidade das poltronas do ônibus \\
\hline & & & Espaço Circulação no ônibus \\
\hline & & & Ventilação no ônibus \\
\hline & & & Suspensão do ônibus \\
\hline & & \multirow{4}{*}{$\begin{array}{c}\text { Estado de } \\
\text { Conservação }\end{array}$} & Mecânica dos veículos \\
\hline & & & Pneus \\
\hline & & & Pinturas dos veículos \\
\hline & & & Poltronas dos veículos \\
\hline & \multirow{11}{*}{ Serviços } & \multirow{4}{*}{$\begin{array}{l}\text { Atendimento } \\
\text { ao Passageiro }\end{array}$} & Educação dos funcionários \\
\hline & & & Facilidades na compra/troca de passagens \\
\hline & & & Rapidez na solução de problemas \\
\hline & & & Divulgação de informações \\
\hline & & \multirow{2}{*}{ Segurança } & Habilidade do motorista na condução do veículo \\
\hline & & & Forma de dirigir do motorista (direção defensiva) \\
\hline & & \multirow{3}{*}{ Horários } & Frequência de horários disponíveis \\
\hline & & & Adequação dos horários disponíveis \\
\hline & & & Respeito aos horários de saída e chegada \\
\hline & & \multirow[t]{2}{*}{ Limpeza } & $\begin{array}{l}\begin{array}{l}\text { Limpeza interna do ônibus (piso, paredes, } \\
\text { poltronas) }\end{array} \\
\end{array}$ \\
\hline & & & Limpeza externa do ônibus \\
\hline \multirow{4}{*}{ Comunidade } & \multirow{4}{*}{$\begin{array}{c}\text { Pontos de } \\
\text { Parada }\end{array}$} & \multirow{4}{*}{$\begin{array}{c}\text { Embarque/ } \\
\text { desembarque }\end{array}$} & $\begin{array}{l}\text { Conforto da infra-estrutura nos locais } \\
\text { embarque/desembarque }\end{array}$ \\
\hline & & & $\begin{array}{l}\text { Estado de conservação da infra-estrutura nos } \\
\text { locais de embarque/desembarque }\end{array}$ \\
\hline & & & Segurança nos locais de embarque/desembarque \\
\hline & & & Limpeza nos locais de embarque/desembarque \\
\hline
\end{tabular}

Fonte: Viticoski et al. (2013).

Figura 1 - Critérios para fuzzificar valores discretos atribuídos aos elementos de avaliação

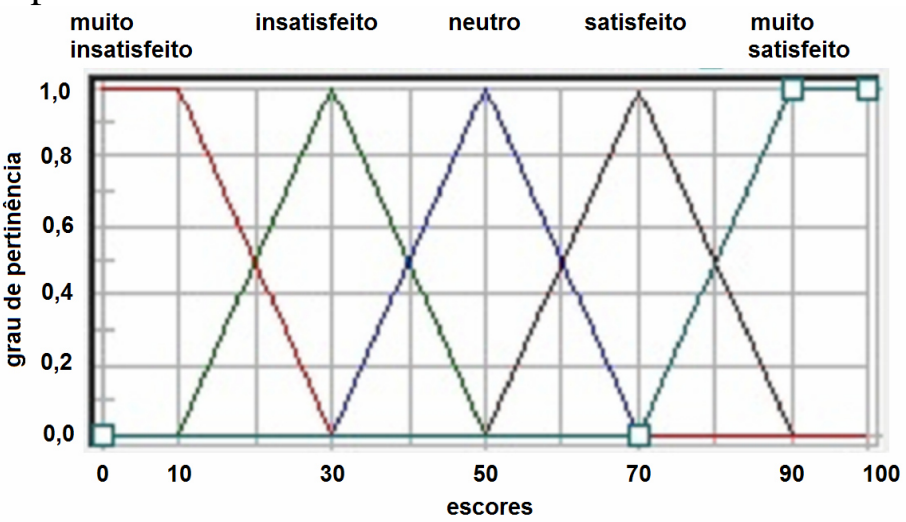

Fonte: Viticoski et al. (2013).

\section{RESULTADOS}

Participaram do estudo 16 indivíduos. Os resultados encontrados pela análise de variância, que considerou 144 observações, são apresentados no quadro 2, mostrando que foram encontradas evidências de que apenas a variável classe tem efeito sobre avaliação da satisfação.

O gráfico (a) da figura 2 mostra que a maior média foi obtida para a classe veículo, seguida da classe serviço, ficando a classe pontos de parada com a pior avaliação média. Um teste Tukey, utilizando nível de significância 5\%, em relação a seta característica identifica dois grupos: 
um deles é formado pelas classes veículo e serviço e o outro pelas classes serviço e pontos de parada.

O gráfico (b) da figura 2 evidencia a pouca diferença entre os escores médios para diferentes métodos de defuzzificação, enquanto que o gráfico (c) desta mesma figura evidencia a mesma distribuição das pontuações médias das classes por método de defuzzificação.

A adequação do modelo encontrado foi considerada satisfatória pelo coeficiente de determinação $(0,888)$ e pela análise gráfica dos resíduos, conforme histograma (a) e gráfico de probabilidade normal (b) apresentados na figura 3.

Quadro 2: Resultados da Análise de Variância

\begin{tabular}{|l|c|c|c|c|c|}
\hline Fonte de variação & SQ & Gl & MQ & F & valor $p$ \\
\hline Método & 164,030 & 2 & 82,015 &, 195 & 0,823 \\
Classe & 3648,752 & 2 & 1824,376 & 4,335 & $0,015^{*}$ \\
Método * Classe & 124,216 & 4 & 31,054 &, 074 & 0,990 \\
Erro & 56812,231 & 135 & 420,831 & & \\
\hline
\end{tabular}

Fonte: análise de dados.

Obs: $\mathrm{SQ}=$ soma dos quadrados; $\mathrm{MQ}=$ média dos quadrados; $\mathrm{gl}$ = graus de liberdade; $\mathrm{F}$ = estatística de teste; valor $\mathrm{p}=$ probabilidade de ocorrência dos dados se não houvesse influência da variável/interação.

(a)

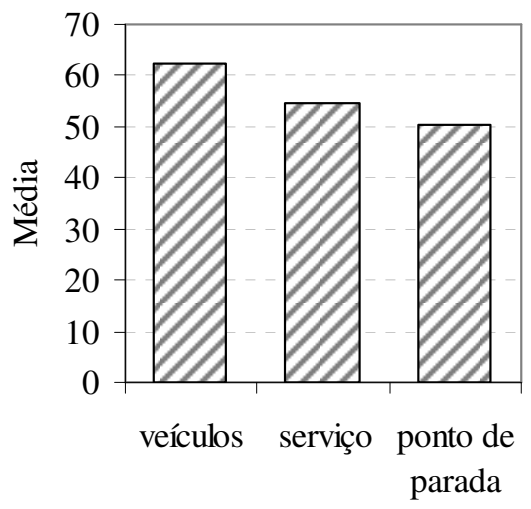

(b)



(c)

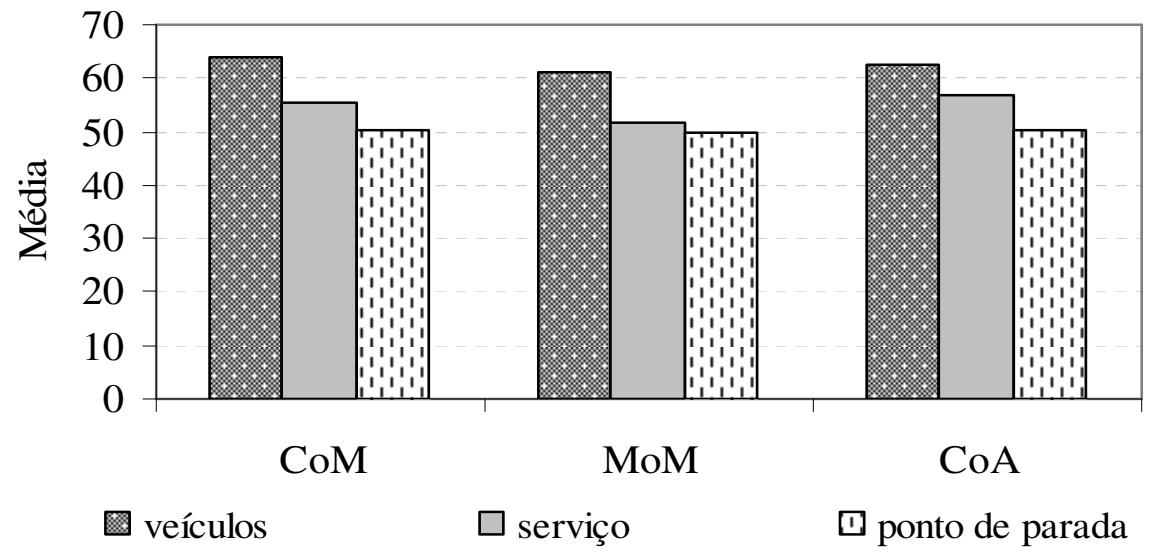

Fonte: análise de dados.

Figura 2 - Escores médios obtidos no instrumento que avalia satisfação 
(a)

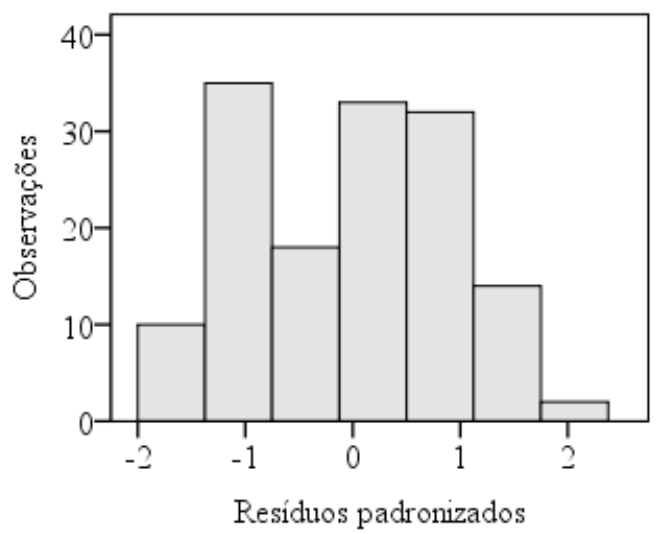

(b)

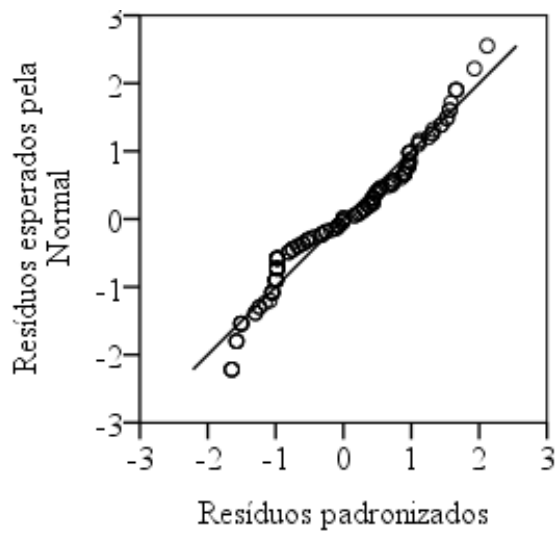

Figura 3 - Histograma (a) e gráfico de probabilidade normal (b) dos resíduos padronizados

$\mathrm{Na}$ análise complementar o coeficiente alfa de Cronbach padronizado foi calculado para os três métodos de defuzzificação considerando as informações obtidas para veículos, serviços e pontos de parada sendo encontrado o resultado 0,789 com o uso do Método da Média dos Máximos (CoM), 0,720 com o uso do Método dos Máximos (MoM) e 0,781 com o uso do Método do Centróide (CoA), sugerindo um pior desempenho do Método dos Máximos na mensuração da satisfação.

\section{CONSIDERAÇÕES FINAIS}

O presente estudo é uma etapa do processo de construção e validação de um instrumento que pretende mensurar a satisfação dos usuários do transporte público municipal e intermunicipal que viabiliza o acesso a um dos campi de uma Instituição de Ensino Superior.

O instrumento desconstrói o construto a ser mensurado - satisfação -, e usa controladores fuzzy para reconstruí-lo. O teste F utilizado na Análise de Variância comparou os resultados obtidos nas três classes de avaliação e não encontrou evidências de diferença entre os resultados obtidos com o emprego de diferentes métodos de defuzzificação. Os coeficientes de confiabilidade foram similares, existindo uma ligeira superioridade no Método da Média dos Máximos. Diante dos resultados encontrados e pela facilidade de implementação, concordando com o método utilizado em Souto (2005), foi selecionado o Método da Média dos Máximos.

Neste estudo exploratório, a análise desenvolvida encontra evidências de diferença nos resultados das avaliações de itens relacionados a veículos, serviços e pontos de parada. A classe melhor avaliada continha itens relacionados a veículos e a pior avaliada a de pontos de parada, o que sugere que a satisfação dos usuários destas linhas de transporte público pode ser aumentada com a implementação de ações para melhorar a qualidade dos locais de embarque e desembarque. Para fazer generalizações destes achados, o tamanho da amostra está sendo ampliado.

\section{AGRADECIMENTOS}

Os autores agradecem a colaboração dos acadêmicos Déborah Cristiane Silva do Carmo; Jéssica Fernandes Araújo e Afonso Ferrer no desenvolvimento de algumas etapas deste estudo.

\section{REFERÊNCIAS BIBLIOGRÁFICAS}

[1] ANTUNES, J. Modelo de avaliação de risco de controle utilizando a lógica nebulosa. Tese (Doutorado) Programa em Pós-Graduação em Contabilidade e Controladoria, Faculdade de Economia, Administração e Contabilidade, Universidade de São Paulo, São Paulo, 2004. 
[2] DUBOIS, D. J.; PRADE, H. M. Fuzzy Sets and systems: Theory and applications. New York: Academic Press, INC. 1980.

[3] MATTOS, V. L. D.; PEREIRA, M. C.; DIMURO, G. P.; ROSA, S. E. Proposta de modelo de avaliação da satisfação de usuários de transporte público usando lógica fuzzy. In: VIII Congresso Nacional de Excelência em Gestão, 2012. Anais..., junho 2012, p.1-14

[4] NAZARI MARYAM ABADI, D.; KHOOBAN, M.H. Design of optimal Mamdani-type fuzzy controller for nonholonomic wheeled mobile robots. Journal of King Saud University - Engineering Sciences, p.1-9, 2013. Disponível em http://dx.doi.org/ 10.1016/j.jksues.2013.05.003

[5] ROSA, S. E. Modelo fuzzy de avaliação de risco para o reconhecimento de Cursos de Graduação, 2012. Dissertação (Mestrado) Programa de Pós-Graduação em Modelagem Computacional, Universidade Federal do Rio Grande, Rio Grande, 2012.

[6] SANAYE, S.; DEHGHANDOKHT, M.; FARTAJ, A. Temperature control of a cabin in an automobile using thermal modeling and fuzzy controller. Applied Energy, v. 97, p. 860-868, 2012. Disponível em http://dx.doi.org/10.1016/j.apenergy.2012.02.078

[7] SIMÕES, M. G.; FRANCESCHETTI, N.N., Bose; BIMAL K. Otimização de um Sistema de Geração de Energia Eólica Através de Controle Fuzzy. Controle \& Automação [online], v.10, n.1, p. 48-58, 1999. Disponível em http://www.sba.org.br/ revista/vol10.htm.

[8] SOUTO, K. C. Sistema Especialista em Lógica Nebulosa para Cálculo em Tempo Real de Indicadores de Desempenho e Segurança na Monitaração de Usinas Nucleares, 2005. Tese (Doutorado) Programa de Pós-Graduação em Engenharia Nuclear, COPPE, Universidade Federal do Rio de Janeiro, Rio de Janeiro, 2005.

[9] VITICOSKI, R. L.; AGREDA, L. M.; MATTOS, V. L. D. O uso de controladores fuzzy na reconstrução de construtos: um estudo exploratório. In: Encontro Nacional de Engenharia de Produção, 2013. Anais... Salvador, 2013, p.1-10.

[10] ZADEH, L. A.. Fuzzy sets. Information and Control, v. 8, n. 3, p. 338-353, 1965. 